\title{
Calorimeter fast simulation based on hit libraries in the LHCb Gauss framework
}

\author{
Matteo Rama $^{1, *}$ and Giacomo Vitali ${ }^{2, * *}$ on behalf of the LHCb collaboration \\ ${ }^{1}$ Istituto Nazionale di Fisica Nucleare sezione di Pisa, Italy \\ ${ }^{2}$ Dipartimento di Fisica, Università di Pisa, Italy
}

\begin{abstract}
In HEP experiments the CPU resources required by Monte Carlo simulations are taking up an ever larger fraction of the total computing power (greater than 75\%). At the same time the pace of performance improvements given by technology is slowing down, so the only solution is a more efficient use of resources. In this context we present the ongoing development of a fast simulation of the LHCb calorimeter based on a point library, which has significant advantages in terms of library size and output accuracy compared to the cell hit library. Preliminary performance studies are presented.
\end{abstract}

\section{Introduction}

During Run 2 the simulation of physics events at $\mathrm{LHCb}$ has taken about $80 \%$ of the distributed computing resources available to the experiment [1]. Nonetheless, for some measurements, the number of events that was possible to simulate has not been sufficient to make the systematic uncertainty associated to Monte Carlo statistics small compared to the main systematic uncertainties [2]. The increase in number of events that will need to be simulated in Run 3 to match the higher luminosity and trigger rate will place an additional burden on the computing resources. To face this situation, it is necessary to develop new ways to significantly increase the speed of the simulation.

A number of fast simulation options are available or under development in $\mathrm{LHCb}$ to complement the standard simulation based on Geant4 [3]. They include simulating only particles from signal decays, reducing the detector geometry, re-using many times the simulated particles not originating from the signal decay under study [4] or using a fully parametric simulation of the detector [5]. All these options are integrated in the existing framework developed for the standard simulation and described in Sec. 2. In this document the ongoing development of a faster calorimeter simulation based on hit libraries is presented.

\section{The LHCb simulation framework}

Two applications, Gauss and Boole, are used to simulate physics events in LHCb. Gauss handles the generation of events and the simulation of particle transport through the detector volume by making use of external generators such as Pythia [6] and the Geant4 toolkit. Boole

\footnotetext{
*e-mail: matteo.rama@pi.infn.it

**e-mail: giacomovitali91@gmail.com
} 

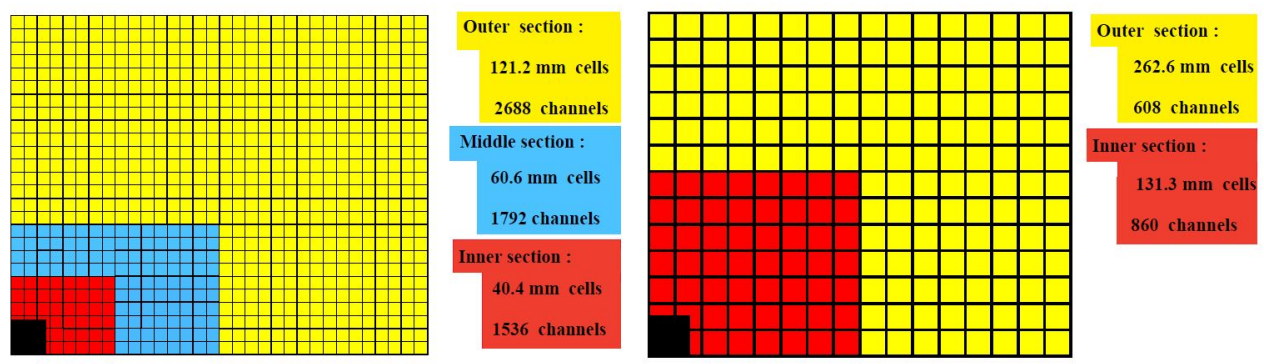

Figure 1. Lateral segmentation (not to scale) of the SPD/PRS and ECAL (left) and the HCAL (right). One quarter of the detector front face is shown. In the left figure the cell dimensions are given for the ECAL.

emulates the detector response (digitisation). Like all other LHCb applications, both are based on the Gaudi software framework [7]. Since the average CPU time spent in the event generation and digitisation phases is an order of magnitude smaller than the time spent by Geant 4 for the particle transport, the effort to make the detector simulation faster must start from the latter.

In a typical minimum bias event about $55 \%$ of the CPU time used by Geant 4 to simulate particle transportation is spent in the calorimeter system, followed by the ring imaging Cherenkov detectors with about $25 \%$. Depending on the Gauss and Geant 4 versions used, $5 \%$ variations of these fractions are possible. Given these numbers, in the effort of developing a faster detector simulation it is natural to start from the calorimeter.

\section{The LHCb calorimeter}

The LHCb detector [8] is equipped with a scintillator pad detector (SPD), a pre-shower detector (PRS), an electromagnetic calorimeter (ECAL) and a hadronic calorimeter (HCAL). The SPD and PRS consist of scintillating pads with a thickness of $15 \mathrm{~mm}$, interspaced with a $2.5 X_{0}$ lead converter. The electromagnetic calorimeter employs a "shashlik" technology of alternating $4 \mathrm{~mm}$ thick scintillators tiles and $2 \mathrm{~mm}$ thick lead plates arranged perpendicular to the beam pipe. The hadron calorimeter is structured in $4 \mathrm{~mm}$ thick scintillator tiles sandwiched between $16 \mathrm{~mm}$ iron sheets arranged parallel to the beam pipe. The four detectors are not longitudinally segmented but adopt a variable lateral segmentation (shown in figure 1) because the hit density varies by two orders of magnitude over the calorimeter surface. A segmentation into three different sections has been chosen for the ECAL and the SPD/PRS, with square cell sizes of approximately 40,60 and $120 \mathrm{~mm}$ in the inner, middle and outer regions, respectively. The HCAL is segmented into an inner and outer region with square cell sizes of approximately 131 and $262 \mathrm{~mm}$, respectively.

\section{Development of a calorimeter fast simulation based on hit libraries}

\subsection{Nature and energy distribution of particles reaching the calorimeter}

To develop the calorimeter fast simulation it is useful to know the properties of the particles from the signal decays of interest and also the approximate nature and energy distribution of 
particles belonging to the rest of the event at the calorimeter entrance. The latter has been studied by simulating a large number of minimum bias events [9] with Gauss and recording all particles reaching the SPD front face. On average about 400 particles per event reach the calorimeter. The distribution of the particle species is summarised in table 1: about $70 \%$ are photons and $15 \%$ are electrons and positrons. The typical energy spectrum is very soft, as it can be seen in table 2 where the fraction of photons below a given energy threshold is shown. For instance, $44 \%$ of photons entering the SPD front face have energies below $8 \mathrm{MeV}$. On the other hand, the energy spectrum of particles from typical decays of interest extends up to about $100 \mathrm{GeV}$ so that the relevant energy range extends over five orders of magnitude.

Table 1. Population at the calorimeter entrance in minimum bias events.

\begin{tabular}{cc}
\hline Particle & Fraction $(\%)$ \\
\hline$\gamma$ & 69.6 \\
$e^{ \pm}$ & 14.9 \\
$\pi^{ \pm}$ & 6.5 \\
$n$ & 5.4 \\
$p / \bar{p}$ & 1.9 \\
$\mu^{ \pm}$ & 0.6 \\
$K^{ \pm}$ & 0.4 \\
\hline
\end{tabular}

Table 2. Fraction of photons at the calorimeter entrance with energy below the given value in minimum bias events.

\begin{tabular}{cc}
\hline Max energy $(\mathrm{MeV})$ & Fraction $(\%)$ \\
\hline 10000 & 99.7 \\
1000 & 92.9 \\
100 & 75.9 \\
32 & 64.3 \\
16 & 55.2 \\
8 & 44.1 \\
4 & 16.0 \\
\hline
\end{tabular}

\subsection{The single-hit generator for very low energy particles}

Most photons with energy below $40 \mathrm{MeV}$ produce one or zero cell hits in the calorimeter. Therefore, the current idea is to use a simple single-hit generator for particles below a given energy threshold and a more sophisticated generator based on hit libraries for higher energy particles. The single-hit generator takes as input a number of pre-built maps, as a function of the energy and the angle of incidence of the particle on the calorimeter front face, parameterising the hit efficiency and the amount of deposited energy in the cell.

\subsection{The cell hit library and its limitations}

The $\mathrm{LHCb}$ calorimeter response is reasonably uniform under a translation of the particle point of incidence of $n$ cells within the same sector (inner, middle or outer), thus simplifying the building of the cell hit library. The remaining degrees of freedom on which the cell hit generation depends are the particle type, energy $(E)$, angle of incidence $(\theta)$, azimuthal angle $(\phi)$ and, to a lesser extent, the projected entrance point $(x, y)$ of the particle inside the cell 
front surface. This constitutes a 6-dimensional cell hit library for each particle type, with the additional complication occurring when a shower is spread over two calorimeter sectors. In this scenario it is not possible to achieve a good performance of the cell hit library output while keeping the library size down to a manageable level.

\subsection{The point library}

The limitations of the cell hit library outlined in the previous section are solved by adopting a solution that we call point library, where collections of almost-point-like energy deposits, as opposed to cell hits, are stored and subsequently translated and rotated according to the incident particle trajectory. The procedure to build the point library is summarised below.

- The transverse area of the cells is divided into small subregions, or "points". A library of deposited energies in the subregions is built by simulating with Geant 4 a number of particles entering the calorimeter with a fixed azimuthal angle $\phi_{0}$ (for instance, $\phi_{0}=90 \mathrm{deg}$ ) and fixed entrance position $\left(x_{0}, y_{0}\right)$. Hence, for a given particle species, only the binnings in the particle energy $E$ and incident angle $\theta$ remain. An example of point collection in the ECAL produced by an incident photon with energy $O(1) \mathrm{GeV}$ is shown in figure 2, top-left plot, where each square of the grid represents the transverse area of an ECAL cell and the colour scale indicates the deposited energy in MeV. In this example the cell has been divided into 25 points.

- Two or more points belonging to the same cell area and with associated energy below a given threshold may be merged locally (energy-weighted barycenter) to simplify the collection, i.e. to reduce the number of points stored in the library. This is exemplified in figure 2, top-right plot.

Once the point library is built, it can be used to simulate the cell hit collection of a particle entering the calorimeter using the following procedure.

- Let us consider a particle entering the calorimeter through the point $(x, y)$ with azimuthal angle $\phi$. A collection of points, among the ones associated to the particle $(E, \theta)$ bin, is randomly selected from the library. Next, the selected points are rotated by $\phi-\phi_{0}$ and translated by $\left(x-x_{0}, y-y_{0}\right)$. This is a key aspect of the point library: the rotated and translated point collection gives a good description of the shower produced by a rotated and translated incident particle. The rotation of the points is exemplified in figure 2, bottom-left plot.

- Finally, the actual calorimeter hits are created by summing the energies of those transformed points which fall into the same cell area. This is shown in figure 2, bottom-right plot, where the colour in the central region of the cell indicates the total deposited energy, in $\mathrm{MeV}$.

This procedure has the additional advantage that a library created from particles entering, for instance, the inner sector of the calorimeter allows to generate cell hits in all sectors, including the inner-middle and middle-outer transition regions. To summarise, for a given particle type, the point library depends only on the energy and angle of incidence of the particle and therefore the number of required parameters is reduced from six to two. The use of a point library, as opposed to a more standard cell hit library, allows to significantly improve the output accuracy for the same size of the library.

\subsection{Preliminary performance studies}

The accuracy of the calorimeter simulation based on point libraries has been tested through the comparison with detailed simulation using photons generated at the calorimeter entrance 

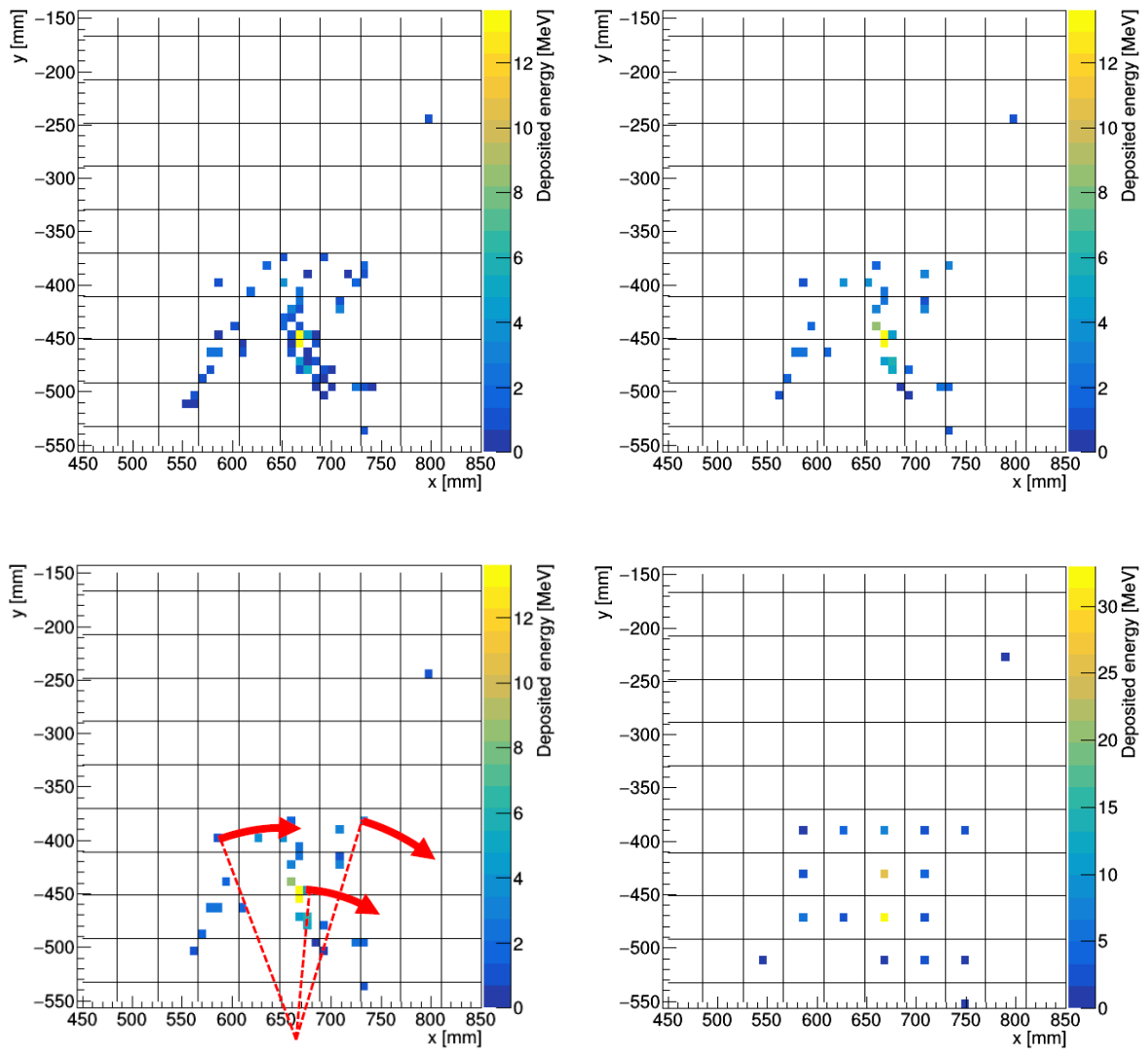

Figure 2. Schematic illustration of the point library operating principle. Each square of the grid represents the transverse area of an ECAL cell and the colour scale indicates the deposited energy in MeV by an incident $1 \mathrm{GeV}$ photon. More details are given in section 4.4.

with various energies and angles of incidence. Three variables relevant for the comparison are the coordinates of the hit cluster centre $x_{c l u s}, y_{\text {clus }}$ and the energy ratio $E_{\text {meas }} / E_{\text {tot }}$, defined as

$$
x_{\text {clus }}=\frac{\sum_{i} E_{i} x_{i}}{\sum_{i} E_{i}}, \quad y_{\text {clus }}=\frac{\sum_{i} E_{i} y_{i}}{\sum_{i} E_{i}}, \quad \frac{E_{\text {meas }}}{E_{\text {tot }}}=\sum_{i} E_{i} / E_{\gamma},
$$

where $x_{i}, y_{i}$ are the $x, y$ positions of the $i^{t h}$ cell centre, $E_{i}$ is the deposited energy and $E_{\gamma}$ is the total energy of the incident particle. In the comparisons shown in figure 3 the fully simulated photons are generated with $E_{\gamma}=2.75 \mathrm{GeV}, \theta=0.26 \mathrm{rad}$ and $\phi=45 \mathrm{deg}$. The point library is created from photons entering the calorimeter inner region with the same value of energy and $\theta$ but a different value of $\phi(90 \mathrm{deg})$ to test the mechanism of the point azimuthal rotation. The top plots compare the $x_{\text {clus }}$ and $y_{\text {clus }}$ distributions in the case where the entrance point of the fully simulated photons coincides with the one used to build the library. In other words, the points of the library have to be rotated by $45 \mathrm{deg}$ but not translated. The bottom plots compare the $y_{\text {clus }}$ and $E_{\text {meas }} / E_{\text {tot }}$ distributions in the case where the fully simulated photons are generated in the outer sector. In this case the points are both rotated and translated but the 
agreement with the full simulation does not worsen, proving that the idea behind the point library works.

The hits for particles with values of $E$ and $\theta$ not coinciding with a library node can be obtained by correcting the points belonging to the closest node. This will be part of the next development steps. Preliminary tests indicate a gain of about 20 in the computing time required to simulate $O(1) \mathrm{GeV}$ photons in the calorimeter using the point library, compared to the Geant4-based simulation.
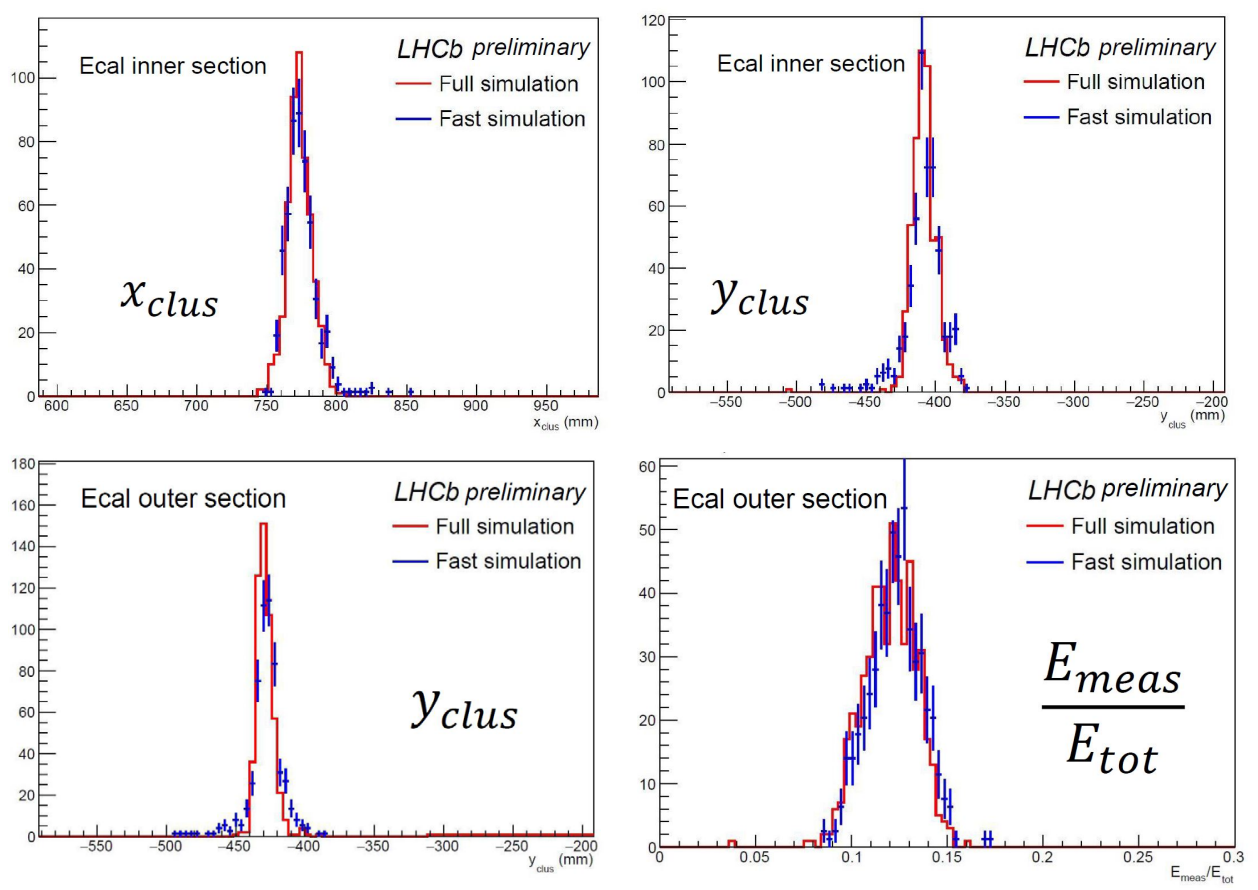

Figure 3. Comparison of photons simulated with Geant4 ("Full simulation") and with the point libraries ("Fast simulation"). Details are given in section 4.5.

\section{Conclusions}

In $\mathrm{LHCb}$ there is an ongoing effort to develop fast simulation alternatives to the nominal detector simulation to face the current and future limitations of CPU resources with respect to the size of the necessary simulated samples. In the detailed simulation based on Geant 4 more than $50 \%$ of the CPU time is spent in the calorimeter system. The development of a faster simulation of the calorimeter based on "point" libraries is underway and the preliminary results in terms of time gain and simulation accuracy are encouraging.

\section{References}

[1] LHCb collaboration, LHCb Upgrade Software and Computing Technical Design Report, CERN-LHCC-2018-007, LHCb-TDR-017. 
[2] LHCb collaboration, R. Aaij et al., Measurement of the ratio of the $B F\left(B^{0} \rightarrow D^{*-} \tau^{+} v_{\tau}\right)$ and $B F\left(B^{0} \rightarrow D^{*-} \mu^{+} v_{\mu}\right)$ branching fractions using three-prong $\tau$-lepton decays, Phys. Rev. Lett. 120 (2018) 171802.

[3] S. Agostinelli et al., (Geant4 Collaboration), Geant4: A simulation toolkit, Nucl. Instrum. Methods Phys. Res., Sect. A 506, 250 (2003); J. Allison et al., (Geant4 Collaboration), IEEE Trans. Nucl. Sci. 53, 270 (2006).

[4] D. Muller et al., ReDecay: A novel approach to speed up the simulation at LHCb, Eur. Phys. J. C 78, 1009 (2018).

[5] B. Siddi, A fully parametric option in the LHCb simulation framework, these proceedings.

[6] T. Sjöstrand, S. Mrenna, P. Skands, A brief introduction to PYTHIA 8.1, Comput. Phys. Commun. 178, 852 (2008).

[7] G. Barrand et al. GAUDI - A software architecture and framework for building HEP data processing applications, Comput. Phys. Commun. 140, 45 (2001).

[8] LHCb collaboration, A. A. Alves Jr. et al., The LHCb detector at the LHC, JINST 3 S08005 (2008).

[9] R. Fields, "Min-Bias and the Underlying Event at the LHC", arXiv:1110.5530. 